BULLETIN OF THE

AMERICAN MATHEMATICAL SOCIETY

Volume 79, Number 4, July 1973

\title{
KRULL DIMENSION-NILPOTENCY AND GABRIEL DIMENSION
}

\author{
BY ROBERT GORDON, THOMAS H. LENAGAN AND J. C. ROBSON \\ Communicated by Hyman Bass, December 27, 1972
}

One property of right noetherian rings and noetherian modules is that they have a Krull dimension in the sense of Rentschler-Gabriel [11]; see below for a definition. This property is considerably weaker than being noetherian. Nevertheless it was shown in [3] and [8] that, if a semiprime ring has a Krull dimension, then it is a right Goldie ring; and it has been conjectured that, in any ring with Krull dimension, nil subrings are nilpotent. Here we describe a proposition by means of which we have established the truth of this conjecture; and we describe other results which follow from it. Several of these are concerned with the connection between the Krull dimension of a module and its "Krull dimension" as defined in [1], which we call its Gabriel dimension. The detailed proofs will appear in [4], [6] and [10].

The proposition, which is basic to the proofs of the results which follow, deals with a module $M$ which has a descending chain of submodules, of order type $\omega$,

$$
M_{0} \supseteq M_{1} \supseteq M_{2} \supseteq \ldots
$$

and an ascending chain of submodules, of order type $\varepsilon$ for some ordinal $\varepsilon$,

$$
B_{1} \subseteq B_{2} \subseteq \cdots \subseteq B_{\delta} \ldots
$$

Let us write $\delta M_{n}=B_{\delta} \cap M_{n}$. From the two chains above, we obtain the following array.

Note that, by the construction of the array, if $\delta \geqq \delta^{\prime}$ then $\delta M_{n} \supseteq \delta^{\prime} M_{n}$ $+\delta M_{n+1}$ for each $n$.

Proposition 1. Suppose that, with the situation as described above, there is a countably infinite sequence of ordinals $\delta_{0}<\delta_{1}<\delta_{2}<\cdots<\varepsilon$ such that, for each $n, \delta_{n+1} M_{n} \supsetneqq \delta_{n} M_{n}+\delta_{n+1} M_{n+1}$. Then the module

$$
\sum_{n=0}^{\infty} \delta_{n+1} M_{n} / \sum_{n=0}^{\infty} \delta_{n} M_{n}
$$

is the infinite direct sum of its nonzero submodules

$$
\delta_{n+1} M_{n}+\sum_{n=0}^{\infty} \delta_{n} M_{n} / \sum_{n=0}^{\infty} \delta_{n} M_{n} .
$$

AMS (MOS) subject classifications (1970). Primary 16A22, 16A46, 16A66. 


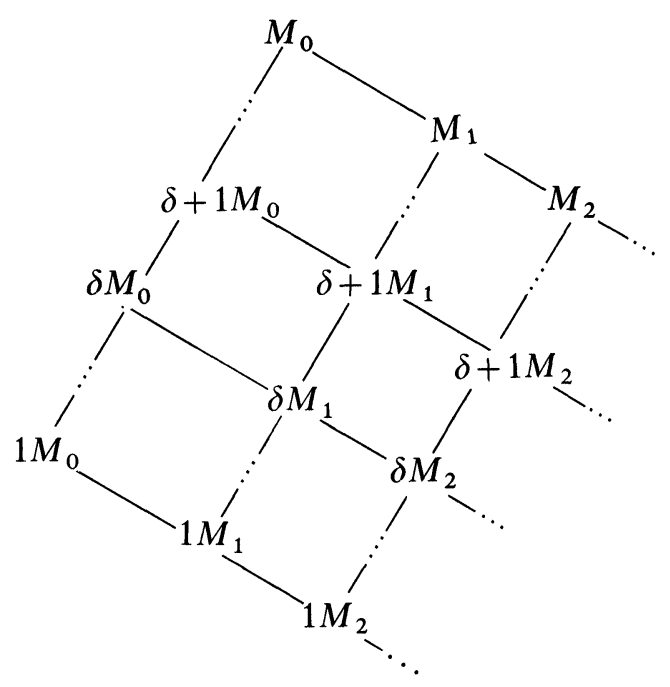

PROOF. This is readily verified.

Before recalling the definition of Krull dimension in the form described in [1], we define recursively certain Serre (épaisse) subcategories $\boldsymbol{A}_{\alpha}^{\prime}$ of an abelian category $\boldsymbol{A}, \alpha$ being an ordinal. $\boldsymbol{A}_{1}^{\prime}$ consists of the artinian objects of $\boldsymbol{A}$; if $\alpha$ is a nonlimit ordinal, $\boldsymbol{A}_{\alpha}^{\prime}$ consists of all objects of $\boldsymbol{A}$ which are artinian in the quotient category $\boldsymbol{A} / \boldsymbol{A}_{\alpha-1}^{\prime}$; and if $\alpha$ is a limit ordinal, $A_{\alpha}^{\prime}=\bigcup_{\beta<\alpha} A_{\beta}^{\prime}$.

If $R$ is a ring and $A$ is the category of right $R$-modules $M$, then the Krull dimension of $M, K \operatorname{dim} M$, is the least ordinal $\alpha$ such that $M \in A_{\alpha+1}^{\prime}$, if such an ordinal exists. Finally, the Krull dimension of $R, K \operatorname{dim} R$, is the Krull dimension of the right $R$-module $R$ again provided it exists.

The usefulness of Proposition 1 in dealing with rings or modules which have a Krull dimension lies in the fact [7] that every homomorphic image of a module with Krull dimension has finite uniform dimension (meaning that it does not contain any infinite direct sum of nonzero submodules).

The first theorem we state is proved in different ways in [4] and [10]. Its validity was conjectured in the original version of [4], and partial results have been obtained in [2], [5] and [9].

THEOREM 2. Nil ideals of a ring with Krull dimension are nilpotent.

This, in conjunction with results in [4] and [12], has the following consequences.

COROLlaRY 3. Let $R$ be a ring with Krull dimension.

(a) Nil subrings of $R$ are nilpotent. 
(b) Each ideal of $R$ contains a product of prime ideals.

(c) $R$ is a right order in a right artinian ring if and only if $R$ satisfies the regularity condition.

(d) $K \operatorname{dim} R=K \operatorname{dim} R / N$ where $N$ is the prime radical of $R$.

The other dimension of concern to us is called the Krull dimension in [1] but here, as in [6], we call it the Gabriel dimension. We start by defining certain full subcategories of a Grothendieck category $\boldsymbol{A}$; but this time they are localizing subcategories $\boldsymbol{A}_{\alpha}$. We let $\boldsymbol{A}_{1}$ be the smallest localizing subcategory containing all objects of $\boldsymbol{A}$ of finite length; if $\alpha$ is a nonlimit ordinal, $\boldsymbol{A}_{\alpha}$ is the smallest localizing subcategory containing the objects of $\boldsymbol{A}$ which have finite length in $\boldsymbol{A} / \boldsymbol{A}_{\alpha-1}$; and if $\alpha$ is a limit ordinal, $\boldsymbol{A}_{\alpha}$ is the smallest localizing subcategory containing $\bigcup_{\beta<\alpha} A_{\beta}$.

Henceforth, we let $\boldsymbol{A}$ denote the category of right $R$-modules for some ring $R$. If $M \in A_{\alpha}$, and $\alpha$ is minimal, then the Gabriel dimension of $M$, $G \operatorname{dim} M$, is defined to be $\alpha$. Note that any module with Krull dimension has a Gabriel dimension and that

$$
G \operatorname{dim} R=\sup \{G \operatorname{dim} M \mid M \in A\}
$$

if either side exists.

COROllary 4. The Gabriel dimension of a ring with Krull dimension cannot be a limit ordinal.

The connection between $G \operatorname{dim} M$ and $K \operatorname{dim} M$ is described in the corollary of the next theorem. The theorem's proof again depends upon Proposition 1 and will appear in [6].

THEOREM 5. Let $M$ be a module with Gabriel dimension and suppose that every homomorphic image of $M$ has finite uniform dimension. Then any chain of submodules of $M$,

$$
M_{0} \supsetneq M_{1} \supsetneq M_{2} \supsetneq \cdots,
$$

such that $G \operatorname{dim} M_{n} / M_{n+1}=G \operatorname{dim} M$ for all $n$ is finite in length.

Corollary 6. (a) A module with Gabriel dimension has Krull dimension if and only if every homomorphic image has finite uniform dimension.

(b) If a module $M$ has Krull dimension then either $G \operatorname{dim} M=K \operatorname{dim} M$ or $G \operatorname{dim} M=K \operatorname{dim} M+1$.

There are examples which show that both possibilities in (b) can occur.

Let $\left\langle\boldsymbol{A}_{\alpha}^{\prime}\right\rangle$ denote the smallest localizing subcategory of $\boldsymbol{A}$ containing $A_{\alpha}^{\prime}$. One easy consequence of Corollary 6 is that if $M$ has Krull dimension and $M \in\left\langle\boldsymbol{A}_{\alpha}^{\prime}\right\rangle$ then $K \operatorname{dim} M \leqq \alpha$. By using a less trivial argument, one can obtain the next result which improves on this. 
TheOREM 7. If a module $M$ has Krull dimension and $M \in\left\langle\boldsymbol{A}_{\alpha+1}^{\prime}\right\rangle$ then $K \operatorname{dim} M \leqq \alpha$.

This has the following consequences.

COROLlary 8. (a) If $R$ is a ring with Krull dimension and $M$ is an $R$-module with Krull dimension then $K \operatorname{dim} M \leqq K \operatorname{dim} R$.

(b) If $M$ is a module with Krull dimension and $\alpha$ is an ordinal then $M$ has a largest submodule of Krull dimension less than or equal to $\alpha$.

The final result we wish to mention concerns the classical Krull dimension of a $\operatorname{ring} R, \operatorname{cl} K \operatorname{dim} R$, which is measured on spec $R$. If $P \in \operatorname{spec} R$ is maximal, set $\kappa(P)=0$; otherwise set $\kappa(P)=\sup (\kappa(Q)+1)$ where $Q$ ranges over $Q \in \operatorname{spec} R, Q \supsetneqq P$. Then cl $K \operatorname{dim} R=\sup _{P \in \operatorname{spec} R} \kappa(P)$.

COROLlaRY 9. If $R$ is a commutative ring with Krull dimension then $K \operatorname{dim} R=\operatorname{cl} K \operatorname{dim} R$.

ProOF. See [4].

\section{REFERENCES}

1. P. Gabriel, Des catégories abéliennes, Bull. Soc. Math. France 90 (1962), 323-448. MR 38 \# 1144.

2. A. W. Goldie and L. W. Small, A study in Krull dimension, J. Algebra (to appear).

3. R. Gordon and J. C. Robson, Semiprime rings with Krull dimension are Goldie, J. Algebra (to appear).

4. — Krull dimension, Mem. Amer. Math. Soc. No. 133 (1973).

5. - Nilpotency in rings with Krull dimension, (unpublished).

6. - The Gabriel dimension of a module, J. Algebra (to appear).

7. G. Krause, On the Krull dimension of left noetherian left Matlis-rings, Math. Z. 118 (1970), 207-214. MR 43 \# 4850.

8. B. Lemonnier, Sur une class d'anneaux definie a partir de la deviation, C. R. Acad. Sci. Paris 274 (1972), 297-299.

9. T. H. Lenagan, Nil ideals in rings with finite Krull dimension, J. Algebra (to appear).

10. - The nil radical of a ring with Krull dimension (to appear).

11. R. Rentschler and P. Gabriel, Sur la dimension des anneaux et ensembles ordonnés, C. R. Acad. Sci. Paris Sér. A-B 265 (1967), A712-A715. MR 37 \#243.

12. L. W. Small, Correction and Addendum: Orders in artinian rings, J. Algebra 4 (1966), 505-507. MR 34 \# 200.

Department of Mathematics, University of Leeds, Leeds, Great Britain

Department of Mathematics, University of Utah, Salt Lake City, Utah 84112

Department of Mathematics, Queen Elizabeth College, london, Great Britain

Current address (Robert Gordon): Department of Mathematics, Temple University, Philadelphia, Pennsylvania 19122 